\title{
Mechanical properties of flax and hemp yarns designed for the manufacturing of geo textiles. Improvement of the resistance to soil born microorganisms
}

\begin{abstract}
Geotextiles are widely used to stabilize river banks from erosion when these ones are restored into vegetal covered areas as mentioned by European regulations. For these applications imported coir (coconut shell fibres) based geotextiles are generally used because coir fibres show a good resistance to soil degradation. In Europe, flax and hemp plants are already grown for textile, building or oil applications. By-products of these industries such as flax tows and short hemp fibres were used to manufacture yarns. The resistance to degradation via the measurement of the mechanical properties of these yarns submitted to enzymatic (cellulase) and microbial attacks (Cellvibrio gandavensis) mimicking soil degradation was evaluated. Large decreases in mechanical properties were observed, even though these ones were still higher than the as received reference coir material. After impregnation by chitosan of the fibres, the tensile properties of the yarns globally remained unchanged after severe attacks. The chitosan acts as a protection against the soil microorganism attacks. As a consequence, flax and hemp by-products could be good candidates for local manufacturing of biodegradable geotextiles.
\end{abstract}

Keywords: flax, hemp, biomaterials, degradability, chitosan, mechanical properties
Volume I Issue 5 - 2017

\author{
Pierre Ouagne,' Sullivan Renouard, ${ }^{2}$ Davina \\ Michel, ${ }^{2}$ Eric Laine ${ }^{2}$ \\ 'ENITarbes, France \\ 2University in Orléans, France
}

Correspondence: Pierre Ouagne, ENI Tarbes, LGP EAI905, F-650 16, Tarbes, France, Tel 330567450I I6, Email pierre.ouagne@enit.fr

Received: March 03, 2017| Published: May 09, 2017

\section{Introduction}

Natural fibre-based geotextiles have found a particular interest for the last two decades as an increasing knowledge of natural fibre properties has been gained. Primary uses of geotextiles are separation or stabilization, drainage, erosion control and reinforcement of road sides or river banks. If plastic based geotextiles were mainly used up to recently, some concerns about their end of life and their impact on the environment led to the design of natural fibre based products. Widelyopen woven textiles, quasi exclusively manufactured manually from coir and jute fibres, are more easily biodegraded at the end of their service life than plastic based materials. However, to be suitable for a geotextiles use, natural fibre materials should have reasonably good mechanical properties, good resistance to biodegradation such as resistance to microbial attacks. ${ }^{1}$

Flax tows from textile breeds, flax fibres from oleaginous breeds and short hemp fibres are by-products of the textile, vegetal oil or building industries respectively. They represent in France large renewable resources that could be used to manufacture geotextiles industrially. From a mechanical point of view, hemp and flax fibres have higher tensile strength, compared to coir fibres..$^{2-4}$ Up to now the highest properties are reported for flax and nettle fibres slightly above hemp..$^{5-12}$ However, their weak point is their low antimicrobial activity. ${ }^{13-15}$ This may be due to the low micro-fibrillar angles observed for flax and hemp (about $10^{\circ}$ and $6^{\circ}$ respectively $)^{16}$ as compared to coir $45^{\circ}$. Indeed, high micro-fibrilar angles have for consequence to reduce the sensitivity to microorganism attack. ${ }^{17}$ So solutions to improve their resistance to microorganisms' degradation should be studied. ${ }^{18,19}$
Chitosan a widespread biodegradable polymer exhibits antimicrobial activity (bacteriostatic and fungistatic) ${ }^{20,21}$ Hamzed et al. ${ }^{22}$ suggest that chitosan impregnation could improve the strength of bagasse fibres. Some investigations on chitosan impregnated textiles, showed a certain dependency of the antimicrobial activity with the chitosan molecular weight. ${ }^{23}$ Renouard et al. ${ }^{24}$ found that chitosan impregnation with low molecular weight is more effective than high molecular weight for the protection of fibres against cellulase and bacteria. Thus, to prevent premature degradations of the bio-based geotextiles, flax and hemp yarns were impregnated by a low molecular weight chitosan and their tensile mechanical properties determined before and after severe enzymatic and bacterial attacks to investigate the potential of these fibres to be manufactured into geotextiles. The measured properties of flax and hemp yarns were compared to the ones of coir yarns which is nowadays the reference on the market. A discussion about the role of chitosan to maintain a good mechanical property level after enzymatic treatment and bacterial attacks is presented in this paper.

\section{Materials and methods}

\section{Materials}

Three different materials were used to study the biodegradation of natural fibre yarns. To study the resistance to degradation of flax tows and hemp fibres, these ones were twisted into yarns, by Groupe Depestele (Le Bocasse, France).$^{25}$ Coir yarns, imported from India, were provided by Ecobiotex (Thizy, France). ${ }^{26}$ The coir yarns are twisted in double yarns Figure 1. The physical properties of the yarns are presented in Table 1. 
Table I Physical and Tensile properties of un-processed flax, hemp and coir yarns

\begin{tabular}{lllll}
\hline & Yarns fineness (tex) & Torque (rd/m) & Tensile strength (MPa) & Young's modulus (MPa) \\
\hline Flax & $4187 \pm 396$ & 64 & $72 \pm 7$ & $759 \pm 40$ \\
Hemp & $4312 \pm 513$ & 62 & $45 \pm 5$ & $788 \pm 28$ \\
Coir & $6172 \pm 1754$ & Double twisted yarn & $14 \pm 2$ & $91 \pm 24$ \\
\hline
\end{tabular}

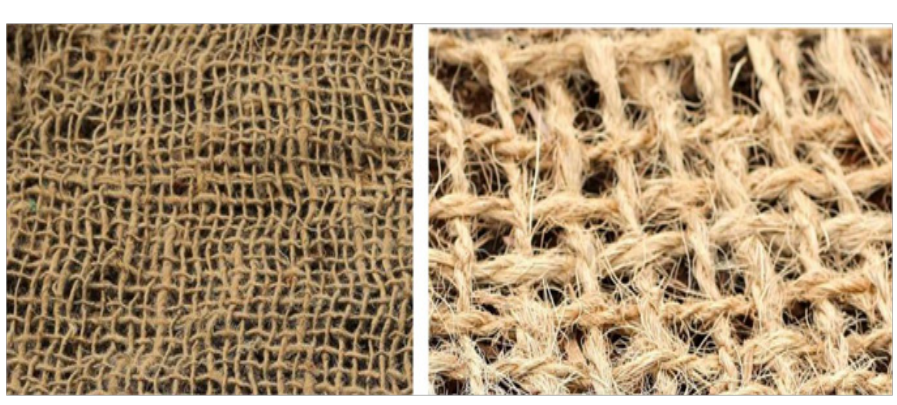

Figure I Jute and Coco based geotextiles: (ECOBIOTEX: www.ecobiotex. $\mathrm{fr})$.

\section{Degradation treatments}

In relation to geotextiles application, mechanical properties of dry fibres were investigated by simulating accelerated soil degradation conditions. For this purpose, enzymatic and microorganism treatments were conducted in optimized conditions. ${ }^{24}$

In this work enzymatic and bacterial degradation were performed as described by Renouard et al. ${ }^{16}$ by using Aspergillus Niger cellulase extract and Cellvibrio gandavensis bacteria. They were respectively purchased from Sigma and $\mathrm{BCCM}^{\mathrm{TM}} / \mathrm{LMG}$ bacteria collection, Gent University (Belgium).

\section{Chitosan impregnation of yarns}

Flax and hemp yarns were submerged into a chitosan solution, prepared with dissolved chitosan powders in $1 \%$ acetic acid at room temperature. Solutions were stirred for 8hours. Yarns were then immerged in chitosan solution under shaking for $16 \mathrm{~h} .{ }^{24}$ The impregnated yarns were then dried in an oven, at $37^{\circ} \mathrm{C}$ during 3 days.

\section{Tensile properties}

Based on the textile standards NF G00-003, ${ }^{27}$ specimens were conditioned at $22^{\circ} \mathrm{C} \pm 2^{\circ} \mathrm{C}$ at room temperature and $65 \% \pm 2 \%$ of relative humidity for 24 hours before starting the tests. Tensile tests were conducted on un-treated, protected and submitted to enzymatic and bacterial attacks flax, hemp and coir yarns, according to the ASTM 2256 standard. ${ }^{28}$ Yarns were weighted, length and diameter measured in order to calculate their physical properties such as cross section and fineness (linear density). All the tensile tests were conducted on an INSTRON 4507 tensile machine with a $5 \mathrm{kN}$ load cell.

\section{Statistical treatments of data}

All data presented in this study are the mean and standard deviation of, at least, 5 independent replicates. Comparative statistical analysis of groups was performed using Student's test. Statistical tests were considered to be significant at $\mathrm{p}<0.05$. Statistical treatments were performed using the EXCELSTAT software.

\section{Results}

\section{Tensile strength}

Tensile strength of un-protected yarns: Average values of tensile strengths of as-received flax, hemp and coir fibres are presented in Table 1 as a comparison basis for the evolution of the tensile properties with different treatments. One can observe that the tensile strength of the flax yarns $(72 \pm 7 \mathrm{MPa})$ is significantly higher than the one of the hemp yarns $(45 \pm 5 \mathrm{MPa})$ and this for equivalent yarn fineness and torque values. A ratio of 1.6 is observed. This is due to the fact that more ligneous residues are part of the yarn structure. The hemp yarn is therefore coarser and the probability to encounter weak zone is higher than for the neater flax yarn. In the meantime, Table 1 also indicates that the tensile strength of flax and hemp yarns is much higher than the one of the reference coco yarn used to elaborate commercial geotextiles $(14 \pm 2 \mathrm{MPa})$. The flax and hemp yarn strengths are respectively 5.1 and 3.2 times higher than the coco yarn one. The ratios become even larger if one compares the relative strength values (strength/fineness) because the fineness of flax and hemp yarns is 1.5 times lower. In this case, the relative flax and hemp strengths are respectively 7.6 and 4.6 times higher than the coco reference value.

When submitted to enzymatic and bacterial environments, the as received flax and hemp yarns show large statistically significant tensile strength decreases when compared to their initial properties. The tensile strength values for enzymatic and microbial degraded flax yarns show respectively a decrease of $33 \%$ and $38 \%$, whereas the equivalent values for hemp yarns are $53 \%$ and $43 \%$. This shows that for both approaches large tensile strength decreases took place. However, in spite of a similar chemical composition, flax presents higher tensile properties than hemp, even after degradation (Figure 2A) (Figure 2E). After degradation by cellulase, the initial ratio of tensile strength (1.6) between the yarns is equal to 2 . It is also very interesting to note that the values of the degraded as received flax and hemp yarns (unprotected) exhibit strength values that are larger than the strength of the non-degraded by enzymatic or bacterially reference coco based yarn. The degraded strength of flax and hemp are respectively larger by factors of about 3 and 2 for flax and hemp respectively.

Tensile strength of chitosan impregnated yarns: Results on mean tensile strength, presented in Figure 2B \& 2F do not show any significant difference between the as received strength and the chitosan treated one. When treated by chitosan, the ratio between the flax and hemp yarns is 1.4 (Figure 2H). The ratio is globally not affected by the addition of the protection layer. The strength values for flax and hemp yarns treated by chitosan are respectively $69 \pm 10 \mathrm{MPa}$ and $49 \pm 5 \mathrm{MPa}$. It is important to note that the addition of the protective layer of chitosan does not almost change the tensile resistance of the flax and hemp yarns. When submitted to enzymatic and microbial attacks, no significant difference can be observed between the chitosan impregnated yarns before and after attacks (Figure 2C) (Figure 2G) for both flax and hemp yarns. This therefore indicates that the chitosan as initially 
expected well prevents the degradation of the tensile resistance of the cellulose rich flax and hemp fibres.

\section{Young's modulus}

Young's modulus of un-protected yarns: Table $1 \&$ Figure 3D indicates that the Young's modulus values for flax $(759 \pm 40 \mathrm{GPa})$ and hemp $(788 \pm 28 \mathrm{GPa})$ as received yarns do not show any statistically significant difference. This is due to the fact that the structure of the yarns is globally equivalent. The fineness of the yarns are relatively close to each other (4187 \pm 396 tex) for flax and (4312 \pm 513$)$ for hemp and the torque almost identical ( $64 \mathrm{rd} / \mathrm{m}$ for flax and $62 \mathrm{rd} / \mathrm{m}$ for hemp). For as received yarns, statistically significant drops of young's modulus are observed after enzymatic and bacterial attacks (Figure 3A) (Figure $3 \mathrm{E})$. For flax, both the attacks result in globally equivalent results $(-45 \%)$ with no statistical difference between the enzymatic and the bacterial degradation. For hemp, bacterial attack seems to be the most damaging $(-66 \%)$ in comparison to the enzymatic one $(-53 \%)$.

It is interesting to note here that the degradation of the untreated as received hemp modulus is more severe than the one of the flax yarns. This follows the same tendency as the one observed for the strength parameter. The enzymatically and bacterially degraded as received flax and hemp yarns (unprotected) exhibit module values that are larger than the ones of the non-degraded by enzymatic or bacterial attack reference coco based yarn (Figure 3A) (Figure 3E). The degraded modulus of flax and hemp are respectively larger by factors of about 4 and 3 for flax and hemp respectively.

Young's modulus of chitosan impregnated yarns: When impregnated by chitosan, the yarn rigidity (Young's modulus) of flax is not statistically affected (Figure 3B). One can however note that a decrease by a factor 1.3 (or a decrease of $27 \%$ ) for the hemp yarn modulus takes place Figure $3 \mathrm{~F}$. When treated by chitosan, the ratio between the flax and hemp yarns module is 1.2 Figure $3 \mathrm{H}$. The addition of chitosan has therefore for effect to reduce the modulus of hemp and not the one of flax. The ratio between the two flax and hemp yarns is therefore affected.

It is important to note that the addition of the protective layer of chitosan does not almost change the modulus of the flax yarns whereas it changes the modulus of the hemp ones Figure 3G. This is probably due to the coarser nature of the hemp yarn. Indeed, the addition of chitosan in a coarser yarn may increase the in homogeneity of the fibrous structure and therefore may decrease its tensile modulus.

\section{Tensile strength (MPa)}

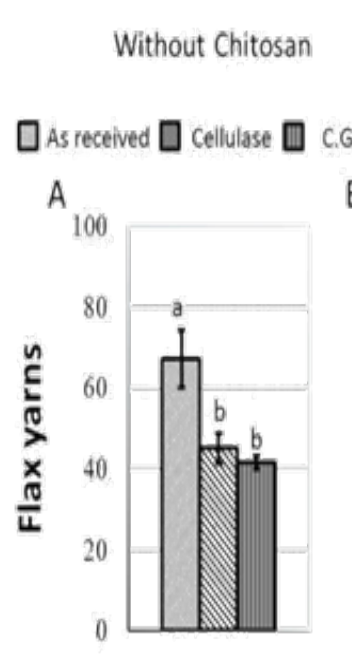

\section{Non-degraded}

With Chitosan

\section{Non-degraded} Flax/Hemp
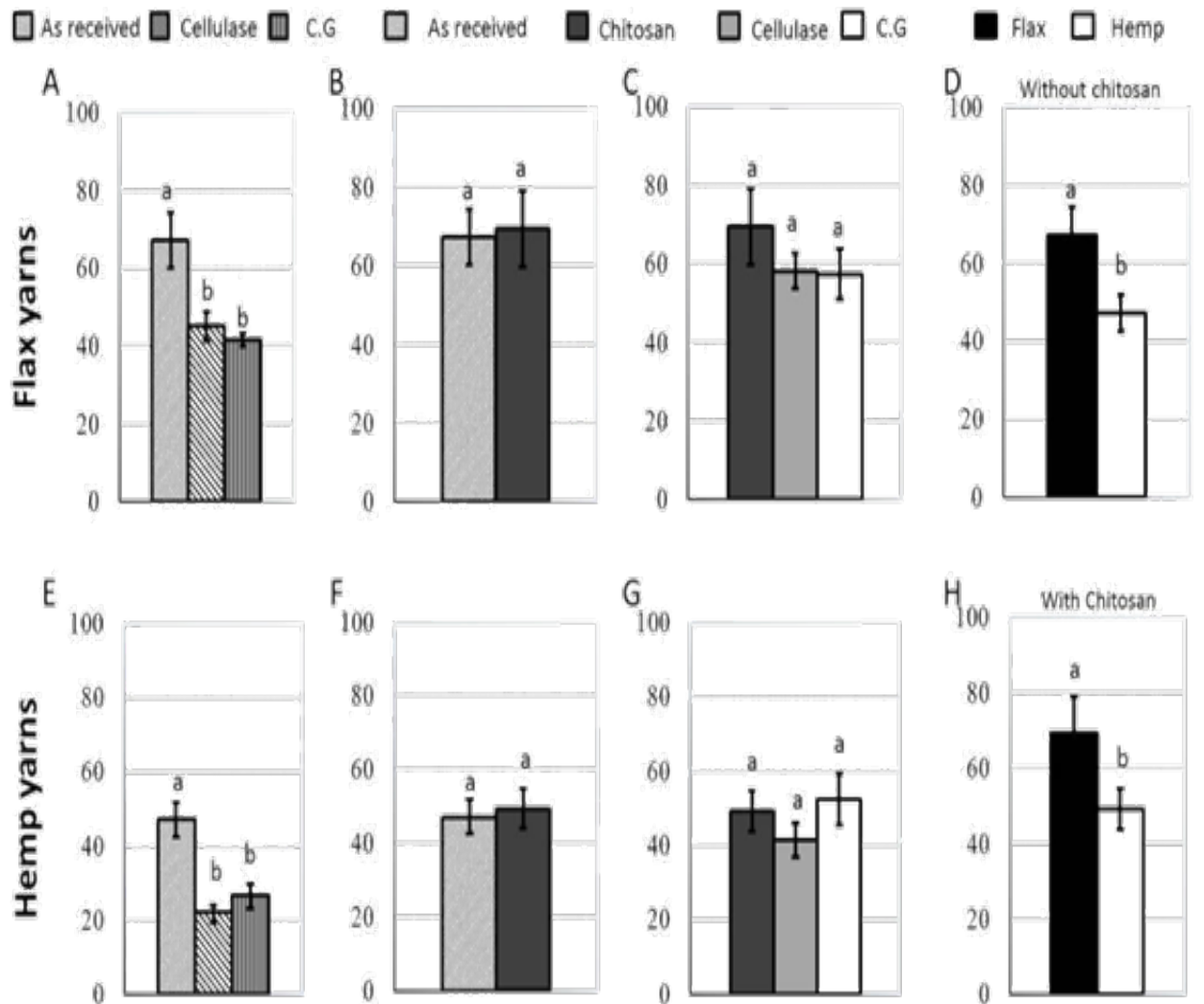

G
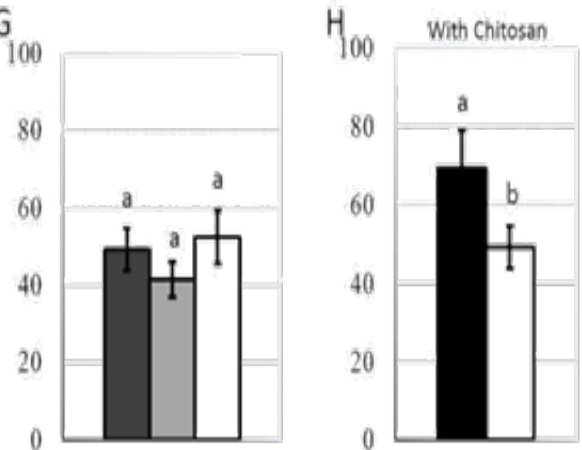

Figure 2 Evolution of tensile strength of flax and hemp yarns for different protection and degradation treatments.

A Degradation of flax yarns by cellulase and Cellvibrio Gandavensis; B Influence of chitosan impregnation for flax yarns tensile strength; C Degradation of flax yarns impregnated by chitosan by cellulase and Cellvibrio Gandavensis; D Tensile strength comparison of flax and hemp yarns; E Degradation of hemp yarns by cellulase and Cellvibrio Gandavensis; F Influence of chitosan impregnation; $G$ Degradation of hemp yarns impregnated by chitosan by cellulase and Cellvibrio Gandavensis; $\mathrm{H}$ Tensile strength comparison of flax and hemp yarns impregnated by chitosan; Measurements associated to different letters differ significantly one from another at $\mathrm{P}<0.05$ using Student test. 


\section{Young's modulus (MPa)}

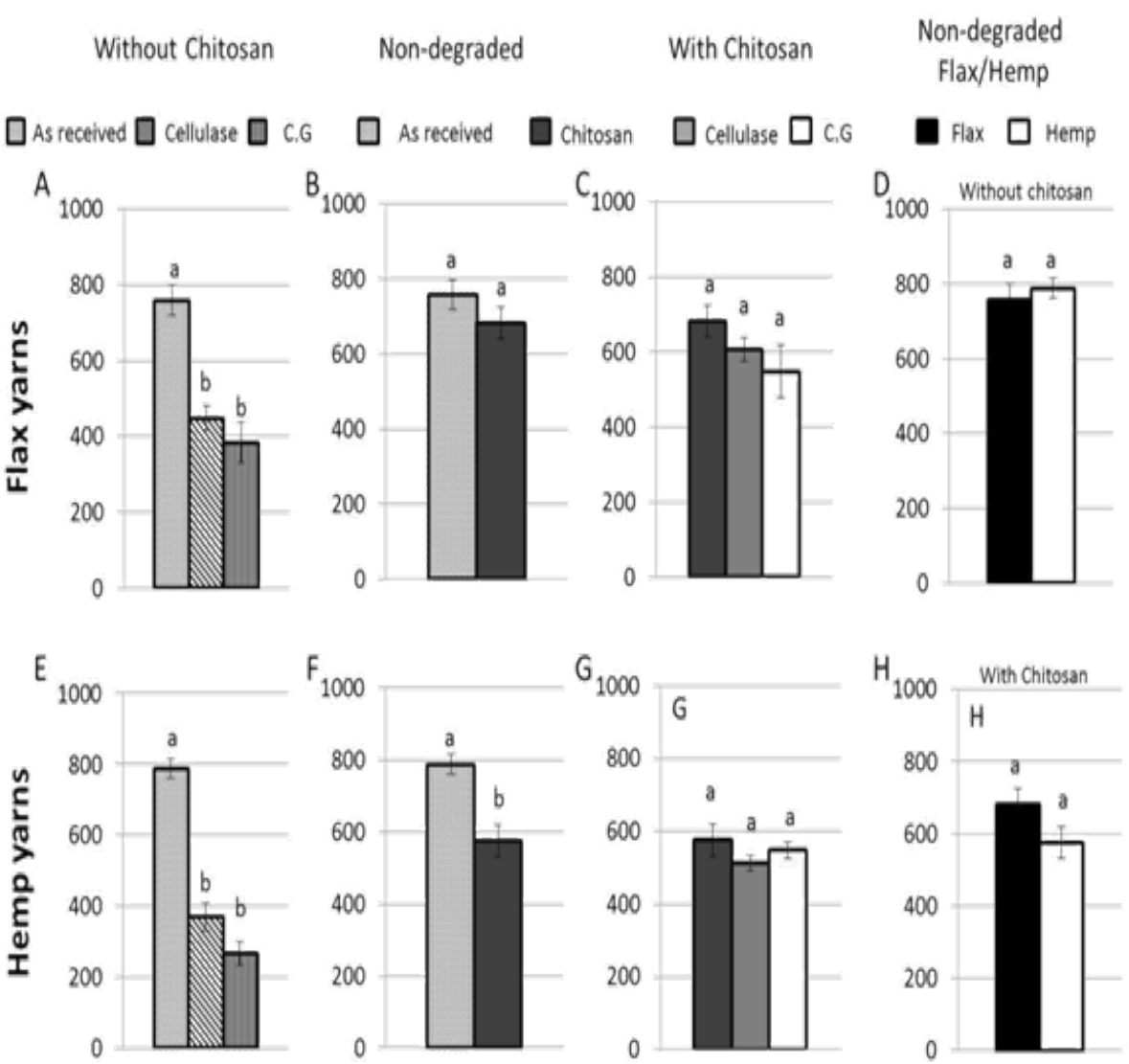

Figure 3 Evolution of young's modulus of flax and hemp yarns for different protection and degradation treatments.

A Degradation of flax yarns by cellulase and Cellvibrio Gandavensis; B Influence of chitosan impregnation for flax yarns Young's Modulus; C Degradation of flax yarns impregnated by chitosan by cellulase and Cellvibrio Gandavensis; D Young's Modulus comparison of flax and hemp yarns; E Degradation of hemp yarns by cellulase and Cellvibrio Gandavensis; F Influence of chitosan impregnation; $G$ Degradation of hemp yarns impregnated by chitosan by cellulase and Cellvibrio Gandavensis; H Young's Modulus comparison of flax and hemp yarns impregnated by chitosan; Measurements associated to different letters differ significantly one from another at $\mathrm{P}<0.05$ using Student test.

\section{Discussion}

From the results presented in Table $1 \&$ Figure 2, tendencies can be extracted. A first one shows that the tensile strength of as received flax yarns are higher than the hemp one Table 1. This is probably due to the fibre/ligneous residues ratio. Indeed, the hemp yarn is coarser, less homogeneous and possesses more ligneous residues than the flax yarn. For the Young's modulus the values are equivalent because this parameter is directly related to the nature of the fibres and the structure of the yarn as both yarns possess globally equivalent fineness and torque parameters. Generally, the strength and the modulus of flax individual fibres are larger than the ones of hemp. This is not due to the composition of the fibres but more to the way the fibres are extracted from the stems. Indeed, the methods used to extract hemp fibres are more aggressive and cause more defects in the fibre structure. However, this work shows that the structure effect is the main parameter that controls the modulus of the flax and hemp yarns. The coarser nature of the hemp yarns does not affect the modulus of as received yarns.

The impregnation by chitosan does not affect the tensile strengths and tensile module of flax and hemp yarns (Figures 2C,2G) (Figures $3 \mathrm{C}, 3 \mathrm{G})$. However, one could have expected that the tensile properties of the protected by chitosan yarns are higher as the as received ones as the chitosan may have played a role of load transmission between the fibres.

When submitted to enzymatic degradation such as cellulase, or bacterial attacks such as Cellvibrio gandavensis the tensile properties of un-protected flax and hemp yarns show respectively large statistically significative decreases in strength and modulus. However, even after the most severe degradations, the tensile properties of flax and hemp yarns are higher than the ones of as received coir reference yarns (3times and twice for flax and hemp tensile strength and 4times and 3times for flax and hemp tensile modulus). One could object that structural differences exist between the single twisted flax and hemp yarns and the double twisted coir ones, but the goal of such comparisons is to establish if the reference coir product performances can be overcome. This is obviously the case and the results presented in this work demonstrate the potential of flax and hemp simple yarn structures for the use in geotextiles products without any protective treatments.

To enhance the resistance to bacterial and enzymatic degradation of flax and hemp fibres, a protection by low molecular weight chitosan was applied for its physical protection and bacteriostatic properties. ${ }^{24}$ 
The results presented in this work show that the protection by chitosan is effective for both types of fibres when submitted to very severe cellulase or bacterial attacks. Globally, the once protected flax and hemp yarns do not present any significative loss of tensile strength and young's modulus. This therefore demonstrates that the protection by chitosan preserves the performances of flax and hemp yarns submitted to severe enzymatic and microbial degradations. This effective protection conferred by chitosan estimated by a mechanical property approach is in a good agreement to the results obtained by Renouard et al. ${ }^{24}$ about the inhibition of the degradation of these fibres by chitosan estimated by analytical methods.

As the mechanical properties of the flax or the hemp yarns of this work are much higher than the reference coir ones and this for yarns containing 1.5 less fibrous matter per yarn, one can conclude that the chitosan protected yarns described in this work can be good candidates for geotextiles applications with the view to replace the imported coir geotextiles. Indeed, this work shows that the mechanical properties of the flax and hemp yarns are much higher than the ones of reference coco yarns (3times and twice for flax and hemp tensile strength and 4 times and 3 times for flax and hemp tensile modulus). If one reports the properties to the fineness of the yarns, the difference in properties would be even larger. The tensile strengths would be 4.5 and 3 times for flax and hemp yarns respectively whereas the tensile moduli would be 6 times and 4.5times larger for flax and hemp protected yarns. This indicates that much lower amounts of fibrous materials can be used to obtain similar properties with flax and hemp yarns to the ones of reference coco yarns. This is particularly interesting for the ecological engineering professionals as it could be possible to use much lighter products for equivalent levels of performance and this from products manufactured locally from local agricultural resources. Transportation of larger geotextiles surfaces for a same mass could be also expected. This would have for effect to reduce the cost of transportation of the products and to make the labour of the end users easier.

\section{Conclusion}

To investigate the potential of geotextiles produced from local resources, flax and hemp yarns were submitted to enzymatic and bacterial degradation protocols using Aspergillus Niger cellulase and Cellvibrio gandavensis bacteria to reproduce some of the potential natural degradation mechanisms in soil. In this work, the impact of enzymatic and bacterial degradations on tensile mechanical properties was investigated on as received yarns produced from by-products of the textile and building industries and on yarns impregnated by low molecular weight chitosan. Even if large relative decreases in mechanical properties were observed after degradation protocols for as-received yarns, these ones remained much higher than the reference product for both flax and hemp yarns. The losses in properties were always higher for hemp than for flax. This is probably due to the coarser nature of the hemp yarn. For the yarns impregnated with protective chitosan, the tensile properties globally remained unchanged after severe degradation attacks despite application of the same degradation protocol. This suggests that the flax and hemp yarns impregnated by low molecular weight chitosan would retain their highest mechanical properties when submitted to soil born microorganisms. Chitosan impregnated flax and hemp yarns could therefore at this state be used to manufacture geotextiles with improved mechanical properties in comparison to the coir reference product. As an example, the modulus of flax yarns reported to their mass are 8 times larger than the reference coco products. This indicates that one could design geotextiles with much lower amounts of fibrous matter, and therefore reduce the cost of the geotextiles by this way but also reduce the transportation costs. As the geotextiles could be much lighter, one could expect that the labour of the end users of the geotextiles such as the ecological engineering profession becomes easier.

\section{Acknowledgements}

The authors would like to thank the Region Centre (France) for their financial support.

\section{Conflict of interest}

Author declares there is no conflict of interest in publishing the article.

\section{References}

1. Kozlowski RM. Handbook of natural fiber processing and applications. 1st ed. Wood head publishing; 2012. p. 1-544.

2. Dechant J. Other long vegetable fibers. In: Lewin M, Pearce EM, editors. Handbook of fibre science and technology. Fibre chemistry. Marcel Dekker, New York, USA; 1998;38(8):507-508.

3. Kostic M, Pejic B, Skundric P. Quality of chemically modified hemp fibers. Biores Technol. 2008;99(1):94-99.

4. Sharma US. Eco friendly and versatile coir geotextiles. Geo synthetics Asia congress, India; 1997.

5. Baley C, Bourmaud A. Average tensile properties of French elementary flax fibers. Materials letters. 2014;122:159-161.

6. Martin N, Davies P, Baley C. Comparison of the properties of scutched flax and flax tow for composite material reinforcement. Industrial Crops \& Products. 2014;61:284-292.

7. Lefeuvre A, Bourmaud A, Morvan C, et al. Elementary flax fibre tensile properties: Correlation between stress-strain behaviour and fibre composition. Industrial Crops \& Products. 2014;52:762-769.

8. Bodros E, Baley C. Study of the tensile properties of stinging nettle fibres (Urtica dioica). Materials letters. 2008;62:2147-2149.

9. Marrot L, Lefeuvre A, Pontoire B, et al. Analysis of the hemp fiber mechanical properties and their scattering(Fedora 17). Industrial Crops and Products. 2013;51:317-327.

10. Duval A, Bourmaud A Augier L, et al. Influence of the sampling area of the stem on the mechanical properties of hemp fibers. Materials letters. 2011;65(4):797-800.

11. Placet V. Characterization of the thermo-mechanical behavior of Hemp fibres intended for the manufacturing of high performance composites. Classical Physics. 2009;40:1111-1118.

12. Dhakal HN, Zhang Z. The use of hemp fibres as reinforcement in composites. Bio fiber reinforcement in composite materials, UK; 2015. p. 86-101.

13. Konczewicz W, Kozlowski RM. Enzymatic treatments of natural fibres. Handbook of Natural Fibres: Processing and applications. UK: Woodhead publishing; 2012. p. 168-184.

14. Borsa J. Antimicrobial natural fibres. Handbook of Natural Fibres: Processing and applications. UK: Wood head publishing; 2012. p. 428-466.

15. Fillat A, Gallardo O, Vidal T, et al. Roncero MB. Enzymatic grafting of natural phenols to flax fibres: development of antimicrobial properties. Carbohydrate Polymers. 2012;87(1):146-152. 
16. Ho Thi TN. Etude de l'influence de la température et de l'humidité sur les propriétés mécaniques entraction des fibres de chanvre et de coco. Quebec University, Canada; 2008. p. 1-234

17. Szostak-Kotowa J.Bio deterioration of textiles. International bio deterioration bio degradation. 2004;53:165-170.

18. Lee HS, Park SH, Lee JH, et al. Antimicrobial and biodegradable PLGA medical sutures with natural grapefruit seed extracts. Materials letters. 2013;95:40-43.

19. Alonso D, Gimeno M, Olayo R, et al. Cross link ink chitosan into UVirradiated cellulose fibers for the preparation of antimicrobial-finished textiles. Carbohydrates polymers. 2009;77:536-543.

20. Lim SH, Hudson SM. Review of chitosan and its derivates as antimicrobial agents and their uses as textile chemicals. J Macromolecular science: part C. Polymer reviews. 2003;43:223-269.

21. Rinaudo M. Chitin chitosan: Properties and applications. Progress in Polymer Science. 2006;31:603-632.
22. Hamzed, Ashori A, Khorasani Z, et al. Pre-extraction of hemicelluloses from Bagasse fibers: Effects of dry-strength additives on paper properties. Ind Crop Prod. 2013;43:365-371.

23. No HK, Park NY, Lee SH, et al. Antibacterial activity of chitosans and chitosan oligomers with different molecular weights. Int $J$ Food Microbial. 2002;74(1-2):65-72.

24. Renouard S, Hano C, Ouagne P, et al. Protection of flax-based yarns against natural soil degradation by chitosan. Materials Letters. 2014;137:269-273.

25. Depestele group, Le Bocasse, France; 2014.

26. Ecobiotex, Thizy Les Bourgs, France.

27. Afnor. Textiles : Atmosphère normale de conditionnement et d'essai. 2nd ed. 2005. p. 6.

28. ASTM. Tensile properties of yarns by the single strength method. American Society for Testing and Materials, USA; 2008. 